\title{
Kesirli telegraf kısmi diferansiyel denklemlerin fark şeması metodu ile nümerik çözümü
}

\author{
Mahmut MODANLI* \\ Harran Üniversitesi Fen-Edebiyat Fakültesi, Matematik Bölümü, Osmanbey Kampüsü, Şanlıurfa. \\ Geliş Tarihi (Recived Date): 01.12.2017 \\ Kabul Tarihi (Accepted Date): 28.02.2018
}

\section{Özet}

Bu çalışmada, özellikle mühendislik, finans, fizik ve sismoloji gibi pek çok bilim dalında uygulamalara haiz başlangıç değer koşullarına sahip kesirli telegraf kısmi diferansiyel denklemi ele alındı. Caputo kesirli kısmi türevli denklemin tanımı vasıtasıyla ele alınan kesirli telegraf kısmi diferansiyel denkleminin sonlu farklardaki ifadesi oluşturuldu. Aynı şekilde, ele alınan denklemin abstract formu ifade edildi. Abstract formda verilen bu denklem için sonlu fark şemaları oluşturuldu. Hilbert uzayı üzerinde tanımlanan norma göre denklemin oluşturulan bu sonlu fark şemalar için kararlılık kestirimleri gösterildi. Kararlılık kestirimini ifade eden Teorem ispatıyla birlikte ifade edildi. Sonlu fark şeması metodu kullanılarak $\alpha=0.1,0.5,0.9$ un farklı değerleri için Caputo kesirli türevi vasıtası ile tanımlanan kesirli telegraf kısmi diferansiyel denkleminin nümerik çözümü elde edildi. Burada, kullanılan örnek problemlerin nümerik çözümleri Matlab programı kullanılarak oluşturuldu. Laplace metodu veya geleneksel metotlar yardımıyla elde edilen tam çözüm ile yaklaşık çözümler mukayese edilerek hata analizi yapıldl. Hata analizi tablosundan elde edilen çıkarsamaya göre önerilen metodun ne kadar etkili ve tutarlı olduğu gözlemlendi.

Anahtar kelimeler: Kesirli telegraf kısmi diferansiyel denklem, kararlılık, fark şeması, hata analizi.

\section{Numerical solution of fractional telegraph partial differential equations by difference scheme method}

\begin{abstract}
In this study, fractional telegraph partial differential equation with initial value condition having applications in physics, engineering, finance, seismology and other disciplines is discussed. By applying definition of Caputo fractional, difference scheme
\end{abstract}

\footnotetext{
*Mahmut MODANLI, mmodanli@harran.edu.tr, https://orcid.org/0000-0002-7743-3512
} 
for fractional telegraph partial differential equation is obtained. The abstract form of the considered equation is also stated. The finite difference schemes of the abstract form for fractional telegraph partial differential equation are constructed. The stability estimates of this finite difference scheme is proved with respect to the norm defined on the Hilbert space. The proof of our main theorem determining the stability estimates is given in detail. By using difference scheme method defined by Caputo fractional derivative, numerical solution of fractional telegraph partial differential equation is obtained for different values of $\alpha=0.1,0.5,0.9$. Numerical solutions of our example is tested by using Matlab programming. Error analysis was performed by comparing approximate solutions with exact solution obtained by Laplace or other traditional methods. It is obvious that the proposed method is effective and consistent according to error analysis.

Keywords: Fractional telegraph partial differential equation, stability, difference schemes, error analysis.

\section{Giriş}

Kesirli diferansiyel denklemler mühendislik, finans, fizik ve sismoloji gibi bilim dallarında pek çok uygulamalara sahiptir [1-3]. Bu diferansiyel denklemler zaman ve uzay değişkenlerine göre çözülebilir [4-6]. Kesirli diferansiyel denklemlerin nümerik çözümleri için farklı metotlar vardır. Matris metodu kullanılarak zaman kesirli adveksiyon dağılım denkleminin karalılığı ve yakınsaklığı çalışıldı [7]. [8] de zaman kesirli diferansiyel difüzyon denklemin yaklaşık çözümü theta metodu yardımıyla hesaplandı. Srivastava, Awasthi ve Tamsir zamana bağlı kesirli mertebeden Caputo hiperbolik telegraf denkleminin nümerik sonuçlarını RDTM (Reduced Differential Transform Method) metoduyla buldu [9]. Ashyralyev ve Dal sonlu fark ve iterasyon metotlarını kullanarak $\alpha=1 / 2$ için kesirli hiperbolik kısmi diferansiyel denkleminin Neumann koşuluna bağlı yaklaşık çözümünü çalıştı [10]. [11] de değişken mertebeden kesirli diferansiyel denklemlerin çözümleri verildi. [12] de Üç boyutlu kesirli evrim denklemi için geriye doğru Euler dönüşümlü yön bindirme fark şeması metodu kullanıldı. [13] de Neumann sınır koşullarıyla kesirli difüzyon denklemlerinin nümerik çözümleri sonlu fark metoduyla hesaplandı. [14] referansında kesirli adi diferansiyel denklemlerin yaklaşık çözümü sonlu fark metoduyla elde edildi. Son olarak, ikinci mertebeden kısmi diferansiyel denklemlerin nümerik çözümü sonlu fark ve reproducing kernel metotlarıyla yapıldı [15].

Bu çalışmada,

$$
\left\{\begin{array}{l}
\frac{\partial^{2} u(t, x)}{\partial t^{2}}+\frac{\partial^{\alpha} u(t, x)}{\partial t^{\alpha}}-\frac{\left(a(x) u_{x}(t, x)\right)}{\partial x}+u(t, x)=f(t, x), \\
0<x<L, 0<t<T, 0 \leq \alpha<1, \\
u(0, x)=r(x), \frac{\partial}{\partial t} u(0, x)=g(x), 0 \leq t \leq T, \\
u\left(t, X_{L}\right)=u\left(t, X_{R}\right)=0, X_{L} \leq x \leq X_{R}
\end{array}\right.
$$


değişken katsayılı kesirli telegraf kısmi diferansiyel denkleminin fark şeması metoduyla nümerik çözümü yapıldı. Değişken katsayılı bu denkleme hem bu metodun ilk olarak uygulanması hemde $\alpha$ nın farklı değerleri için yaklaşık çözümlerinin bulunması bu çalışmayı farklı kılar.

Şimdi kesirli analizin bazı temel tanımlarını verelim.

1.1. Tanım Gamma fonksiyonu bütün $z \in R$ için

$\Gamma(z)=\int_{0}^{\infty} e^{-t} t^{z-1} d t$

ile tanımlanır.

1.2. Tanım Zamana bağlı $\alpha$ ıncı dereceden $D_{t}^{\alpha} u(t, x)$ Caputo kesirli türevi $n-1<$ $\alpha<n$ için

$D_{t}^{\alpha} u(t, x)=\frac{\partial^{\alpha} u(t, x)}{\partial t^{\alpha}}=\frac{1}{\Gamma(n-\alpha)} \int_{0}^{t} \frac{1}{(t-p)^{\alpha-n+1}} \frac{\partial^{\alpha} u(p, x)}{\partial p^{\alpha}} d p$

ve $\alpha=n \in N$ için

$D_{t}^{\alpha} u(t, x)=\frac{\partial^{\alpha} u(t, x)}{\partial t^{\alpha}}=\frac{\partial^{n} u(t, x)}{\partial t^{n}}$

olarak tanımlanır.

\section{Fark şeması metodunun kurulması ve kararlılığı}

[16] referansındaki metot kullanılarak (1) denklemi

$$
\left\{\begin{array}{l}
\mathrm{u}^{\prime \prime}(\mathrm{t})+\mathrm{D}_{\mathrm{t}}^{\alpha}(\mathrm{t})+\mathrm{Au}(\mathrm{t})=\mathrm{f}(\mathrm{t}), \quad 0<t<T, \\
\mathrm{u}(0)=\varphi, \quad \mathrm{u}^{\prime}(0)=\Psi, \quad 0 \leq \mathrm{t} \leq \mathrm{T}
\end{array}\right.
$$

formunda yazılabilir. Burada, $f(t)=f(t, x), a(x) \geq a>0$ belirli fonksiyonlardır ve $A, H=L_{2}[0, T]$ Hilbert uzayında

$A u(x)=-\frac{\left(a(x) u_{x}\right)}{\partial x}+u(x)$

ile

$D(A)=\left\{u(x): u(x), u_{x}(x),\left(a(x) u_{x}\right)_{x} \in H=L_{2}[0, T], u(0)=u(T)=0\right\}$

bölgesinde tanımlanan bir self-adjoint pozitif operatördür. 
$x$ ekseni için $h=\frac{L}{M}$ ve $y$ ekseni için $\tau=\frac{T}{N}$ düzgün aralıkları verilsin. Bu taktirde, $n=1,2, \ldots, M$ için $x_{n}=X_{L}+n h$, ve $k=1,2, \ldots, N$ için $t_{k}=k \tau$ dır. (3) formülünün hesaplanması için birinci dereceden fark şeması yaklaşımı

$D_{\tau}^{\alpha} u_{k} \cong s_{\alpha, \tau} \sum_{j=1}^{k} w_{j}^{(\alpha)}\left(u_{k-j+1}-u_{k-j}\right)$

dır. Burada $s_{\alpha, \tau}=\frac{\tau^{-\alpha}}{\Gamma(2-\alpha)}$ ve $w_{j}^{(\alpha)}=(j)^{1-\alpha}-(j-1)^{1-\alpha}$ dir. [17] makalesinde metot kullanılırsa (5) denklemi

$\frac{\partial^{\alpha} u\left(t_{k}, x_{n}\right)}{\partial t^{\alpha}}=s_{\alpha, \tau}\left[w_{1} u_{n}^{k}-w_{k} u_{n}^{0}+\sum_{j=1}^{k-1}\left(w_{k-j+1}^{(\alpha)}-w_{k-j}^{(\alpha)}\right) u_{n}^{j}\right.$

olarak yazılabilir. (4) denklemi için birinci dereceden fark şeması ve (5) formülü kullanilırsa

$\left\{\begin{array}{l}\frac{u_{k+1}-2 u_{k}+u_{k-1}}{\tau^{2}}+D_{\tau}^{\alpha} u_{k}+A u_{k}=f_{k}, \\ f_{k}=f\left(t_{k}\right), t_{k}=k \tau, 1 \leq k \leq N-1, \\ u_{0}=\varphi, \frac{u_{1}-u_{0}}{\tau}=\Psi,\end{array}\right.$

denklemi bulunur. Bu denklem

$\left(\frac{1}{\tau^{2}}\right) u_{k-1}-\left(\frac{2}{\tau^{2}}-A\right) u_{k}+\left(\frac{1}{\tau^{2}}\right) u_{k+1}=F_{k}$

olarak yeniden yazılabilir. Burada $F_{k}=f_{k}-D_{\tau}^{\alpha} u_{k}$ dir.

(8) probleminin bir çözüme sahip olduğu ve aşağıdaki formülün sağlandı̆̆ı [18] den bilinmektedir.

$u_{k}=\frac{R \tilde{R}\left(R^{k-1}-\tilde{R}^{k-1}\right)}{\tilde{R}-R} u_{0}+\frac{\tilde{R}^{k}-R^{k}}{\tilde{R}-R} u_{1}+\sum_{m=1}^{k} \frac{\tau^{2} R \tilde{R}}{(\tilde{R}-R)}\left(\tilde{R}^{k-1}-R^{k-1}\right) F_{m}$

burada

$R=\frac{2-\tau^{2} A+\tau^{2} A^{1 / 2} B^{1 / 2}}{2}, \quad \tilde{R}=\frac{2-\tau^{2} A-\tau^{2} A^{1 / 2} B^{1 / 2}}{2}$

dir. Burada $B=A-\frac{4}{\tau^{2}}$ dir.

$R_{1}=\frac{(I-R)^{2}}{\tilde{R}-R}, R_{2}=-\frac{(I-\tilde{R})^{2}}{\tilde{R}-R}, R_{3}=\frac{\tilde{R}-I}{\tilde{R}-R}, R_{4}=\frac{I-R}{\tilde{R}-R}, R_{5}=R \tau^{2} \frac{\tilde{R}-I}{\tilde{R}-R}, R_{6}=-\tilde{R} \tau^{2} \frac{R-I}{\tilde{R}-R}$

dir.

2.1. Lemma Aşağıdaki eşitsizlikler doğrudur. 
i) $\quad\|R\|_{H} \leq 1,\|\tilde{R}\|_{H} \leq 1$,

ii) $\quad\left\|R_{1}\right\|_{H} \leq M(\tau),\left\|R_{2}\right\|_{H} \leq M(\tau)$,

iii) $\quad\left\|R_{3}\right\|_{H} \leq M(\tau),\left\|R_{4}\right\|_{H} \leq M(\tau)$,

iv) $\quad\left\|R_{5}\right\|_{H} \leq M(\tau),\left\|R_{6}\right\|_{H} \leq M(\tau)$.

Burada $M, \tau$ ya bağlı bir sayıdır. Lemma'nın ispatı

$c(\tau)=\frac{R+\tilde{R}}{2}=1-\frac{A \tau^{2}}{2}$,

$s(\tau)=B^{-1 / 2} \frac{R-\tilde{R}}{2 i}=\frac{A \tau^{2}}{2 i}$

denklemleri ve bu denklemlerden de elde edilen

$R=c(\tau)+i B^{\frac{1}{2}} S(\tau), \quad \tilde{R}=c(\tau)-i B^{\frac{1}{2}} S(\tau)$

formülleri kullanılarak kolayca yapılabilir.

(7) fark şeması denkleminin kararlılığı ile ilgili aşağıdaki teoremi verelim.

2.1. Teorem $A \geq \frac{4}{\tau^{2}}$ şartının sağlandığını kabul edelim. Bu durumda birinci mertebeden (7) doğruluk fark şeması denklemi için aşağıdaki kararlılık kestirimleri sağlanır.

$$
\begin{aligned}
& \underbrace{\max }_{1 \leq k \leq N}\left\|\frac{u_{k+1}-2 u_{k}+u_{k-1}}{\tau^{2}}\right\|_{H}+\underbrace{\max }_{1 \leq k \leq N}\left\|A u_{k}\right\|_{H} \\
& \leq M[\|\varphi\|_{H}+\|\Psi\|_{H}+\left\|f_{1}\right\|_{H}+\underbrace{\max }_{1 \leq k \leq N}\left\|\frac{f_{k+1}-f_{k}}{\tau}\right\|_{H}] .
\end{aligned}
$$

Burada $M, 1 \leq k \leq N$ için $\varphi, \Psi, f_{1}$ ve $\tau$ ya bağlı olmayan bir sayıdır.

\section{İspat.}

$u_{1}=\varphi+\tau \Psi$ olduğu göz önüne alınıp (9) denkleminde yerine yazılırsa

$u_{k}=\frac{(1-R) R^{k-1}-\tilde{R}^{k-1}(1-\tilde{R})}{\tilde{R}-R} \varphi+\tau \frac{\tilde{R}^{k}-R^{k}}{\tilde{R}-R} \Psi+\frac{\tilde{R}^{k-1}-R^{k-1}}{(\tilde{R}-R)} \tau^{2} \mathrm{f}_{1}$

$+\tau^{2} \sum_{m=2}^{k} \frac{R \tilde{R}}{(\tilde{R}-R)}\left(\tilde{R}^{k-1}-R^{k-1}\right) f_{m}-\sum_{m=1}^{k} \frac{\tau^{2} R \tilde{R}}{(\tilde{R}-R)}\left(\tilde{R}^{k-1}-R^{k-1}\right) D_{\tau}^{\alpha} u_{m}$

elde edilir. (11) formülünden $u_{k-j+1}$ ve $u_{k-j}$ değerleri bulunup (5) formülünde yerine yazilırsa 


$$
\begin{aligned}
& D_{\tau}^{\alpha} u_{k}=\sum_{j=1}^{k} s_{\alpha, \tau} w_{j}^{(\alpha)}\left[\left(R_{1} R^{k-j}+R_{2} \tilde{R}^{k-j}\right) \varphi+\left(R_{3} \tilde{R}^{k-j}+R_{4} R^{k-j}\right) \Psi\right. \\
& +\left(R_{5} R^{j-1}+R_{6} \tilde{R}^{j-1}\right) f_{1}+\sum_{m=1}^{j-1}\left(R_{5} \tilde{R}^{j-m-1}+R_{6} R^{j-m-1}\right)\left(f_{m+1}-f_{m}\right) \\
& \left.-\sum_{m=1}^{j}\left(R_{5} R^{j-m}+R_{6} \tilde{R}^{j-m}\right) D_{\tau}^{\alpha} u_{m}\right]
\end{aligned}
$$

bulunur. (12) denkleminden

$$
\begin{gathered}
\left\|D_{\tau}^{\alpha} u_{k}\right\|_{H} \leq \sum_{j=1}^{k} s_{\alpha, \tau} w_{j}^{(\alpha)}\left[\left(\left\|R_{1}\right\|_{H \rightarrow H}\left\|R^{k-j}\right\|_{H \rightarrow H}+\left\|R_{2}\right\|_{H \rightarrow H}\left\|\tilde{R}^{k-j}\right\|_{H \rightarrow H}\right)\|\varphi\|_{H}\right. \\
+\left(\left\|R_{3}\right\|_{H \rightarrow H}\left\|\tilde{R}^{k-j}\right\|_{H \rightarrow H}+\left\|R_{4}\right\|_{H \rightarrow H}\left\|R^{k-j}\right\|_{H \rightarrow H}\right)\|\Psi\|_{H}+\left(\left\|R_{3}\right\|_{H \rightarrow H}\left\|\tilde{R}^{k-j}\right\|_{H \rightarrow H}\right. \\
+\left(\left\|R_{5}\right\|_{H \rightarrow H}\left\|R^{j-1}\right\|_{H \rightarrow H}+\left\|R_{6}\right\|_{H \rightarrow H}\left\|\tilde{R}^{j-1}\right\|_{H \rightarrow H}\right)\left\|f_{1}\right\|_{H} \\
+\tau \sum_{m=1}^{j-1}\left(\left\|R_{5}\right\|_{H \rightarrow H}\left\|\tilde{R}^{j-m-1}\right\|_{H \rightarrow H}+\left\|R_{6}\right\|_{H \rightarrow H}\left\|R^{j-m-1}\right\|_{H \rightarrow H}\right)\left\|f_{m+1}-f_{m}\right\|_{H} \\
\left.+\tau \sum_{m=1}^{j}\left(\left\|R_{5}\right\|_{H \rightarrow H}\left\|R^{j-m}\right\|_{H \rightarrow H}+\left\|R_{6}\right\|_{H \rightarrow H}\left\|\tilde{R}^{j-m}\right\|_{H \rightarrow H}\right)\left\|D_{\tau}^{\alpha} u_{m}\right\|_{H}\right]
\end{gathered}
$$

elde edilir. Buradan da

$\left\|D_{\tau}^{\alpha} u_{k}\right\|_{H} \leq \frac{\tau^{-\alpha}}{\Gamma(2-\alpha)} \sum_{j=1}^{k} w_{j}^{(\alpha)}\left[\|\varphi\|_{H}+\|\Psi\|_{H}+\left\|f_{1}\right\|_{H}+\sum_{m=1}^{j-1}\left\|f_{m+1}-f_{m}\right\|_{H}\right.$

$+\tau \underbrace{\max }_{1 \leq k \leq N}\left\|D_{\tau}^{\alpha} u_{k}\right\|_{H}] M$

yazılabilir. İntegral eşitsizlik özelliği kullanılarak

$\underbrace{\max }_{1 \leq k \leq N}\left\|D_{\tau}^{\alpha} u_{k}\right\|_{H} \leq M[\|\varphi\|_{H}+\|\Psi\|_{H}+\left\|f_{1}\right\|_{H}+\underbrace{\max }_{1 \leq k \leq N-1}\left\|\frac{f_{k+1}-f_{k}}{\tau}\right\|_{H}]$

ve

$\left\|\sum_{m=1}^{k}\right\| D_{\tau}^{\alpha} u_{m}\left\|_{H}\right\|_{H} \leq M[\|\varphi\|_{H}+\|\Psi\|_{H}+\left\|f_{1}\right\|_{H}+\underbrace{\max }_{1 \leq k \leq N-1}\left\|\frac{f_{k+1}-f_{k}}{\tau}\right\|_{H}]$

bulunur. Üçgen eşitsizliği özelliğinden

$\left\|\frac{u_{k+1}-2 u_{k}+u_{k-1}}{\tau^{2}}+A u_{k}\right\|_{H} \leq M[\|\varphi\|_{H}+\|\Psi\|_{H}+\left\|f_{1}\right\|_{H}+\underbrace{\max }_{1 \leq k \leq N-1}\left\|\frac{f_{k+1}-f_{k}}{\tau}\right\|_{H}]$ 
yazılabilir.

(16) denkleminden de

$\underbrace{\max }_{1 \leq k \leq N}\left\|\frac{u_{k+1}-2 u_{k}+u_{k-1}}{\tau^{2}}\right\|_{H} \leq M[\|\varphi\|_{H}+\|\Psi\|_{H}+\left\|f_{1}\right\|_{H}+\underbrace{\max }_{1 \leq k \leq N-1}\left\|\frac{f_{k+1}-f_{k}}{\tau}\right\|_{H}]$

elde edilir.

(10), (13) ve (14) formülleri kullanılarak

$\underbrace{\max }_{1 \leq k \leq N}\left\|u_{k}\right\|_{H} \leq M[\|\varphi\|_{H}+\|\Psi\|_{H}+\left\|f_{1}\right\|_{H}+\underbrace{\max }_{1 \leq k \leq N-1}\left\|\frac{f_{k+1}-f_{k}}{\tau}\right\|_{H}]$

olduğu görülür.

(14), (16) ve (17) kestirimleri kullanılarak (10) kestiriminin doğru olduğu görülür.

\section{Nümerik uygulamalar}

Giriş bölümünde verilen (1) değişken katsayılı kesirli telegraf denkleminde $a(x)=$ $x^{2}$ alınıp bu denklemde Cauchy-Euler dönüşümü uygulanırsa sabit katsayılı kesirli telegraf denklemi elde edilir. Şimdi kesirli telegraf kısmi diferansiyel denkleminin nümerik sonuçlarını görmek için aşağıdaki örneği araştıralım.

\section{1. Örnek}

$$
\left\{\begin{array}{l}
\frac{\partial^{2} u(t, x)}{\partial t^{2}}+\frac{\partial^{\alpha} u(t, x)}{\partial t^{\alpha}}-\frac{\partial^{2} u(t, x)}{\partial x^{2}}-\frac{\partial u(t, x)}{\partial x}+u(t, x)=f(t, x) \\
f(t, x)=\left(6 t+6 \frac{t^{3-\alpha}}{\Gamma(4-\alpha)}+2\left(t^{3}+1\right)\right) \sin x-\left(t^{3}+1\right) \cos x \\
0<x<\pi, 0<t<1,0 \leq \alpha<1 \\
u(0, x)=\sin x, \frac{\partial}{\partial t} u(0, x)=0,0 \leq t \leq 1 \\
u(t, 0)=u(t, \pi)=0,0 \leq x \leq \pi
\end{array}\right.
$$

denklemi veriliyor. (18) denkleminin tam çözümünün $0 \leq \alpha<1$ aralığındaki bütün $\alpha$ değerleri için

$$
u(t, x)=\left(t^{3}+1\right) \sin x
$$

olduğu [19] referansındaki yöntem ve Laplace metodu kullanılarak bulunabilir.

$\mathrm{Bu}$ son denklem için fark şeması 


$$
\left\{\begin{array}{l}
\frac{u_{n}^{k+1}-2 u_{n}^{k}+u_{n}^{k-1}}{\tau^{2}}+s_{\alpha, \tau} \sum_{j=1}^{k} w_{j}^{(\alpha)}\left(u_{n}^{k-j+1}-u_{n}^{k-j}\right) \\
-\frac{u_{n+1}^{k}-2 u_{n}^{k}+u_{n-1}^{k}}{h^{2}}-\frac{u_{n+1}^{k}-u_{n-1}^{k}}{2 h}+u_{n}^{k}=f_{n}^{k}, \\
f_{n}^{k}=f\left(t_{k}, x_{n}\right)=\left(6 t_{k}+6 \frac{t_{k}^{3-\alpha}}{\Gamma(4-\alpha)}+2\left(t_{k}^{3}+1\right)\right) \sin x-\left(t_{k}^{3}+1\right) \cos x_{n}, \\
1 \leq k \leq N-1,1 \leq n \leq M-1, \\
u_{n}^{0}=\sin x_{n}, \frac{u_{n}^{1}-u_{n}^{0}}{\tau}=0,0 \leq n \leq N, \\
u_{0}^{k}=u_{M}^{k}=0,0 \leq k \leq M,
\end{array}\right.
$$

şeklindedir. Bu problemi çözmek için Modifiye Gauss Eliminasyon metodu uygulandi. Birinci mertebeden doğruluk fark şeması uygulanarak $N=M=40,80,160$ için Tablo 1. elde edildi. $u(t, x)$ tam çözümü ve $u\left(t_{k}, x_{n}\right)$ nümerik çözüm olmak üzere,

$E_{M}^{N}=\underbrace{\max }_{1 \leq k \leq N-1,1 \leq n \leq M-1}\left|u\left(t_{k}, x_{n}\right)-u_{n}^{k}\right|$

formüllü kullanılarak nümerik hesaplamalar bulundu. Bulunan bu nümerik sonuçlar aşağıdaki tabloda verilmiştir.

Tablo 1. (19) denkleminin hata analizi.

\begin{tabular}{|c|c|c|c|}
\hline$\alpha$ & $N=M=40$ & $N=M=80$ & $N=M=160$ \\
\hline 0.1 & 0.0224 & 0.0114 & 0.0058 \\
\hline 0.5 & 0.0223 & 0.0118 & 0.0061 \\
\hline 0.9 & 0.0119 & 0.0066 & 0.0036 \\
\hline
\end{tabular}

\section{Sonuçlar ve tartışma}

Bu çalışmada, Caputo tipi değişken katsayılı kesirli telegraf denklemi ele alındı. Kesirli telegraf denklemi için fark şemaları oluşturuldu. Bulunan bu fark şemaları için kararlılık kestirimleri yapıldı. Değişken katsayılı kesirli telegraf denklemi CauchyEuler Metodu yardımıyla sabit katsayılı kesirli telegraf denklemine dönüştürülerek fark şeması metodu kullanılarak nümerik sonuçları bulundu. Matlab programı yardımıyla bulunan bu nümerik sonuçlar tam çözümle karşılaştırılarak hata analizi yapıldı. Aynı çalışma Riemann-Liouville tipindeki kesirli telegraf kısmi diferansiyel denklemler için de yapılabilir. 


\section{Kaynaklar}

[1] Celik, C. ve Duman M., Crank-Nicholson method for the fractional equation with the Riezs fractional derivative, Journal of computational physics, 231, 17431750, (2012).

[2] Gorial, I.I., Numerical methods for fractional reaction-dispersion equation with Riesz space fractional derivative, Engineering. and Techology Journal, 29, 709-715, (2011).

[3] Jafari, H., Gejii, V.D., Solving linear and nonlinear fractional diffusion and wave equations by adomian decomposition, Applied Mathematics and Computation, 180,488-497, (2006).

[4] Karatay, I., Bayramoglu, S.R. ve Sahin, A., Implicit difference approximation for the time fractional heat equation with the nonlocal condition, Applied Numerical Mathematics, 61, 1281-1288, (2011).

[5] Su, L., Wang, W. ve Yang, Z., Finite difference approximations for the fractional advection-diffusion equation, Physics Letters A., 373, 4405-4408, (2009).

[6] Tadjeran, C., Meerschaert, M. M. ve Scheffler, H. P., A Second-order Accurate Numerical Approximation for the Fractional Diffusion Equation, Journal of Computational Physics, 213, 205-213, (2006).

[7] Karatay, I., Kale, N. ve Bayramoglu Erguner, S. R., Stability and Convergence of a Finite Partial Diferential Equations by Matrix Method, International Mathematical Forum, 9, 1757-1765, (2014).

[8] Aslefallah, M., Rostamy, D. ve Hosseinkhani, K., Solving time-fractional differential diffusion equation by theta method, International Journal of Applied Mathematics and Mechanics, 2, 1-8, (2014).

[9] Srivastava, V.K., Awasthi, M K. ve Tamsir, M., RDTM solution of Caputo time fractional-order hyperbolic telegraph equation, AIP advances, 3, 032142, 1-11, (2013).

[10] Ashyralyev, A. ve Dal, F., Finite Difference and Iteration Methods for Fractional Hyperbolic Partial Differential Equations with the Neumann Condition, Discrete Dynamics in Nature and Society, 2012, 1-15, ( 2012).

[11] Akgul, A., Inc, M. ve Baleanu, D., On solutions of variable-order fractional differential equations, An International Journal of Optimization and Control: Theories \& Applications, 7(1), 112-116, (2017).

[12] Chen, H., Xu, D., Cao, J. ve Zhou, J., A backward Euler alternating direction implicit difference scheme for the three-dimensional fractional evolution equation, Numerical Methods for Partial Differential Equation, (2017).

[13] Szekeres, B. ve Izsák, F., A finite difference method for fractional diffusion equations with Neumann boundary conditions, Open Mathematics, 13(1), pp. -. Retrieved 21 Feb. (2018), from doi:10.1515/math-2015-0056.

[14] Changpin, L. ve Fanhai, Z., The Finite Difference Methods for Fractional Ordinary Differential Equations, Numerical Functional Analysis and Optimization, 34, 2, 149-179, (2013).

[15] Modanli, M. ve Akgul, A., On solutions to the second-order partial differential equations by two accurate methods, Numerical Methods for Partial Differential Equations, 1-15, (2017).

[16] Ashyralyev, A. ve Modanli, M., An operator method for telegraph partial differential and difference equations, Boundary Value Problems, 2015(1), 1-17, 2015). 
[17] Karatay, I., Bayramoglu, S.R. ve Sahin, A., A new difference scheme for time fractional heat equation based on the Crank-Nicholson method, Fractional Calculus and Applied Analysis, 16, 892-910, (2013).

[18] Ashyralyev, A. ve Sobolevskii, P.E., New Difference Schemes for Partial Differential Equations, Birkhauser, Verlag, Basel, Boston, Berlin, (2004).

[19] Kumar, S., A new analytical modelling for fractional telegraph equation via Laplace transform, Applied Mathematical Modelling, 38, 3154-3163, (2014). 